\title{
Evaluación de cinco residuos avícolas como fuentes de nitrógeno mineral disponible
}

\author{
Evaluation of five poultry residues as sources of available mineral nitrogen \\ Luz Palomino ${ }^{1 *}$, Ruby Vega ${ }^{1}$, Coralí Lara², Luis Gomero ${ }^{3}$, Sady García ${ }^{*}$
}

\begin{abstract}
RESUMEN
La producción orgánica en el Perú se ha incrementado significativamente en la última década, aumentando con ello la demanda de fuentes nitrogenadas de alta disponibilidad, que satisfagan los requerimientos de los cultivos. La mineralización del nitrógeno en cinco residuos avícolas: estiércol de gallina ponedora (EP), estiércol de gallina reproductora (ER), estiércol con restos de pollo (ERP), harina de pluma (HP) y lodo de flotación (LF), fue estudiada con la finalidad de evaluar su potencial como fertilizantes orgánicos de rápida liberación. Los residuos fueron aplicados a dosis de $0.33 \%$ (p/p) en tubos que contenían arena lavada, e incubados aeróbicamente a $25^{\circ} \mathrm{C}$ durante 120 días. Las fracciones nítrica $\left(\mathrm{N}_{-} \mathrm{NO}_{3}^{-}\right)$y amoniacal $\left(\mathrm{N}-\mathrm{NH}_{4}{ }^{+}\right)$fueron extraídas de los tubos y determinadas por colorimetría después de 0, 30, 60, 90 y 120 días de incubación. El potencial de mineralización fue calculado como la máxima cantidad de $\mathrm{N}$ mineral $\left(\mathrm{N}_{-} \mathrm{NH}_{4}{ }^{+}+\mathrm{N}^{-} \mathrm{NO}_{3}{ }^{-}\right)$liberado durante la incubación. La tasa de mineralización fue máxima en los 30 primeros días y continuó hasta los 120 días para la harina de pluma y hasta 90 días en las demás fuentes. Los residuos mostraron un potencial de mineralización de entre 12 y $70 \%$. La harina de plumas y los estiércoles de gallina ponedora y reproductora presentaron los mayores potenciales de mineralización de $\mathrm{N}$ y son fuentes promisorias para su empleo en la fertilización de cultivos orgánicos.
\end{abstract}

Palabras clave: Potencial de mineralización, nitrógeno disponible, harina de pluma, agricultura orgánica.

\begin{abstract}
The production of organic crops in Peru has significantly increased during the last decade, thus increasing the demand of organic sources with high availability of nitrogen, suitable to satisfy crop requirements. The mineralization of nitrogen in five residues of poultry production: layer manure (EP), reproducer manure (ER), broiler manure with chicken corpses (ERP), feather meal (HP) and floatation sludge $(L F)$, was studied with the aim of evaluating their potential as rapid-release organic fertilizers. The residues were applied at a rate of $0.33 \%(\mathrm{p} / \mathrm{p})$ in centrifuge tubes containing washed sand, and aerobically incubated at $25{ }^{\circ} \mathrm{C}$ during 120 days. Mineral $\mathrm{N}$ fractions: nitrate $\left(\mathrm{N}-\mathrm{NO}_{3}^{-}\right)$and ammonium $\left(\mathrm{N}-\mathrm{NH}_{4}{ }^{+}\right)$were extracted from tubes and determined by colorimetric procedures at 0, 30, 60, 90 and 120 days of incubation. Nitrogen mineralization potential was calculated as the maximum amount of mineral $\mathrm{N}\left(\mathrm{N}-\mathrm{NH}_{4}{ }^{+}+\mathrm{N}-\mathrm{NO}_{3}^{-}\right)$released during the incubation. Nitrogen mineralization rate was the highest at 30 days and continued until 120 days in feather meal, and until 90 days in the other sources. The organic residues showed $N$ mineralization potential of 12 y 70\%. Feather meal, layer manure and reproducer manure showed the highest $N$ mineralization potential, and are promissory sources to be used as fertilizers in organic crops.
\end{abstract}

Key words: Mineralization potential; available nitrogen; feather meal; organic agriculture.

\section{Introducción}

La producción de cultivos orgánicos en el Perú se ha incrementado significativamente en la última década, hasta ubicarse como el quinto país con mayor área de producción orgánica en América Latina en el año 2017 con 315500 ha (FiBL e
IFOAM, 2019). El nitrógeno (N) es a menudo el nutriente más limitante para la producción de cultivos orgánicos, especialmente en aquellos altamente demandantes. El guano de isla es el abono más empleado en la agricultura orgánica en el Perú, pero su disponibilidad a través del año es estacional. Otras fuentes, como el compost o

1 Departamento Académico de Suelos, Universidad Nacional Agraria La Molina, Lima-Perú.

2 San Fernando S.A., Lima-Perú.

3 Facultad de Ciencias Ambientales, Universidad Científica del Sur, Lima-Perú.

* Autor de correspondencia: sjgarciab@lamolina.edu.pe Av. La Molina s/n, La Molina, Lima-Perú.

Fecha de Recepción: 21 de abril, 2019.

Fecha de Aceptación: 29 Agosto, 2019. 
los residuos de cultivos, son usadas comúnmente porque son relativamente fáciles de adquirir. Sin embargo, su velocidad de mineralización puede ser demasiado lenta como para satisfacer los requerimientos de $\mathrm{N}$ mineral $\left(\mathrm{NH}_{4}{ }^{+}\right.$y $\left.\mathrm{NO}_{3}{ }^{-}\right)$de los cultivos. Los residuos de la industria avícola (estiércoles, plumas, sangre, etc.) son fuentes alternativas ricas en nitrógeno, pero recomendar su uso extendido requiere del conocimiento del aporte real de nitrógeno en forma mineral.

La efectividad de los residuos orgánicos como proveedores de $\mathrm{N}$ disponible puede estimarse mediante procesos de incubación que permiten cuantificar la máxima cantidad de $\mathrm{N}$ mineral y la tasa de mineralización de $\mathrm{N}$ (Cerrato et al., 2007). Diversas investigaciones se han enfocado en evaluar la capacidad de los subproductos avícolas como fuente de $\mathrm{N}$ mineralizable. Tyson y Cabrera (1993) encontraron valores de 5,8 y $40 \%$ de $\mathrm{N}$ mineral en estiércol compostado y no compostado, respectivamente. Li y Li (2014) detectaron tasas de mineralización de hasta $21 \%$ para el $\mathrm{N}$ en estiércol de aves incubado. Agehara y Warncke (2005) hallaron entre 37 y $45 \%$ de $\mathrm{N}$ mineral liberado, en tanto que Abbasi y Khalig (2016) reportaron hasta $48 \%$. La harina de plumas mostró $65 \%$ de mineralización de nitrógeno en 60 días (Hadas y Kautsky, 1994; Hartz y Johnstone, 2006), en tanto que la harina de sangre, aproximadamente $66 \%$ (Hartz y Johnstone, 2006) en 140 días.

La hipótesis descriptiva planteada para esta investigación es que los residuos de la industria avícola poseen $\mathrm{N}$ mineralizable suficiente para proveer $\mathrm{N}$ a los cultivos. El estudio busca evaluar, a través del proceso de mineralización in vitro, la aptitud de cinco residuos avícolas: estiércol de gallina ponedora (EP), estiércol de gallina reproductora (ER), harina de pluma (HP), estiércol con restos de pollo (ERP) y lodo de flotación (LF), como proveedores de $\mathrm{N}$ mineral sostenible, cuantificando el $\mathrm{N}$ mineral liberado y la tasa de mineralización de $\mathrm{N}$.

\section{Materiales y Métodos}

\section{Residuos avícolas}

Los cinco residuos experimentales fueron obtenidos a partir de la materia orgánica en la crianza de aves de la empresa avícola San Fernando (Lima, Perú). El estiércol de gallina ponedora (EP) es retirado de las jaulas semanalmente y secado al aire.
El estiércol de gallina reproductora (ER) mezclado con cama de cascarilla de arroz es removido cada tres meses y secado al aire. Ambos residuos fueron colectados en la granja productora de huevos ubicada en el distrito de Cerro Azul, provincia de Cañete. El estiércol con restos de pollo (ERP) consistió en una mezcla de excreta de pollo con los cadáveres de aves provenientes de la mortandad en granja y compostada por más de tres meses, y fue colectado en la planta de abonos orgánicos en el distrito de Huacho, provincia de Huaura. La harina de pluma (HP) es el material hidrolizado de las plumas de aves, sometido a altas temperaturas para hacerlo más fácilmente degradable por microorganismos (El Boushy et al., 1990), y fue obtenida en la planta de beneficio de aves en el distrito de Chancay, provincia de Huaral. El lodo de flotación (LF) es el sólido suspendido (grasas, ceras, sangre, parte de vísceras) proveniente del tratamiento de aguas residuales y efluentes líquidos de la mencionada planta.

Las muestras fueron secadas a $70^{\circ} \mathrm{C}$ durante 48 horas y molidas para pasar una malla de $1 \mathrm{~mm}$. El pH fue determinado en una pasta saturada de la fuente respectiva empleando agua destilada. El nitrógeno fue determinado por el método de micro-Kjeldahl modificado (Yuen y Pollard, 1953). Posteriormente, muestras de cada fuente orgánica fueron atacadas mediante digestión vía húmeda empleando solución nítrico-perclórica (5:1). El contenido de fósforo en los digestos obtenidos fue determinado mediante colorimetría con el método amino-naftol-sulfónico, en tanto que los contenidos de potasio y calcio fueron determinados por espectrofotometría de absorción atómica. El nitrógeno amoniacal y nítrico $\left(\mathrm{N}_{-} \mathrm{NH}_{4}{ }^{+}\right.$ y $\mathrm{N}^{-\mathrm{NO}_{3}}{ }^{-}$) fue determinado por extracción con $\mathrm{KCl}$ $1 \mathrm{~N} \mathrm{y} \mathrm{K}_{2} \mathrm{SO}_{4} 0,34 \mathrm{M}$, respectivamente, y posterior colorimetría.

Las características químicas de los residuos se muestran en el Tabla 1. El contenido de nitrógeno en los estiércoles (EP, ER y ERP) varió entre 2,0 y $3,8 \%$. Si bien estos contenidos son frecuentes en los estiércoles, son bastante menores a los esperables considerando la dieta de las gallinas ponedoras y reproductoras. Es posible que el manejo del estiércol influya en la pérdida parcial del nitrógeno en estas fuentes. El lodo de flotación (LF) y la harina de pluma (HP) mostraron el mayor contenido de nitrógeno; sin embargo, la HP presenta un tenor menor al reportado por Li y $\mathrm{Li}$ (2014). Los valores de $\mathrm{N}$ amoniacal y nítrico de los estiércoles son similares a los registrados por Li y Li (2014). 
Tabla 1. Características de los residuos avícolas sometidos a incubación.

\begin{tabular}{|c|c|c|c|c|c|c|c|c|}
\hline \multirow{2}{*}{ Residuo } & \multirow{2}{*}{$\begin{array}{c}\mathrm{pH} \\
\left(\mathrm{H}_{2} \mathrm{O} 1: 1\right)\end{array}$} & \multirow{2}{*}{$\mathrm{C} / \mathrm{N}$} & $\mathrm{N}$ & $\mathrm{P}_{2} \mathrm{O}_{5}$ & $\mathrm{~K}_{2} \mathrm{O}$ & $\mathrm{CaO}$ & $\mathrm{N}-\mathrm{NO}_{3}^{-}$ & $\mathrm{N}-\mathrm{NH}_{4}{ }^{+}$ \\
\hline & & & \multicolumn{6}{|c|}{$\mathrm{g} \mathrm{kg}^{-1}$} \\
\hline Estiércol de ponedoras & 7,29 & 14,5 & 21,0 & 52,2 & 39,1 & 113,8 & 0,49 & 3,87 \\
\hline Estiércol de reproductoras & 8,07 & 19,5 & 20,3 & 39,6 & 35,7 & 76,2 & 0,34 & 2,38 \\
\hline Estiércol con restos de pollo & 5,33 & 12,0 & 37,6 & 224,6 & 14,8 & 295,6 & 0,17 & 2,35 \\
\hline Harina de pluma & 4,93 & 4,0 & 133,1 & 5,3 & 1,8 & 3,5 & 0,09 & 2,18 \\
\hline Lodo de flotación & 5,48 & 8,0 & 61,9 & 28,4 & 1,1 & 12,9 & 0,23 & 5,09 \\
\hline
\end{tabular}

\section{Incubación}

El experimento de mineralización de $\mathrm{N}$ bajo condiciones de incubación in vitro se realizó en el Laboratorio de Análisis de Suelos, Plantas, Aguas y Fertilizantes de la Facultad de Agronomía de la Universidad Nacional Agraria La Molina (LASPAF-UNALM) en 2016. Porciones de $50 \mathrm{~g}$ de arena de cuarzo previamente lavada y tamizada a través de una malla de $1 \mathrm{~mm}$ fueron colocadas en tubos de centrifugación de $50 \mathrm{ml}$ de capacidad. Las muestras orgánicas (tamizadas a $1 \mathrm{~mm}$ ) fueron aplicadas en los tubos a razón de $165 \mathrm{mg}$ de peso seco, equivalentes a 70, 440,67, 124, y $204 \mathrm{mg}$ $\mathrm{kg}^{-1}$ de $\mathrm{N}$ orgánico para EP, HP, ER, ERP y LF, respectivamente. Tubos con arena sin fuente orgánica fueron usados como control. Las mezclas fueron homogeneizadas y humedecidas al 50\% de su máxima capacidad de retención empleando agua destilada (Johnson et al., 2012). Un tubo de cada tratamiento fue colocado en frascos de plástico de 11 de capacidad y se instalaron 25 frascos. Cinco frascos fueron extraídos en cada tiempo de evaluación. Para mantener la humedad constante, se agregaron $100 \mathrm{ml}$ de agua en el fondo de cada frasco. Posteriormente los frascos fueron cerrados herméticamente e incubados a $25^{\circ} \mathrm{C}$ durante 120 días. El $\mathrm{N}$ mineral liberado de los cinco residuos y el control fueron evaluados rutinariamente en cinco oportunidades $(0,30,60,90$ y 120 días $)$ mediante muestreo destructivo. En cada evaluación se empleó un diseño completo al azar con seis tratamientos y cinco repeticiones.

\section{Determinación de nitrógeno mineral}

Cinco tubos de cada muestra orgánica tamizada fueron removidos de la incubación, cada 30 días durante 120 días, para realizar las determinaciones químicas (N amoniacal y nítrico). En cada determinación se analizaron también cinco tubos que contenían arena sin adición de materia orgánica como testigo. La concentración inicial fue tomada en el día 0. El nitrógeno amoniacal $\left(\mathrm{N}-\mathrm{NH}_{4}{ }^{+}\right)$ fue determinado por extracción con $\mathrm{KCl} 1 \mathrm{~N}$ y posterior colorimetría empleando fenol básico. El contenido de nitrógeno nítrico $\left(\mathrm{N}^{-\mathrm{NO}_{3}}{ }^{-}\right)$fue determinado por extracción con $\mathrm{K}_{2} \mathrm{SO}_{4} 0,34 \mathrm{M}$ y posterior colorimetría empleando ácido salicílico. El nitrógeno mineral total fue calculado como la suma de las dos fracciones anteriores.

\section{Cálculos}

El N mineral liberado de la mezcla (arena + residuo) en cada tiempo de evaluación (t) fue calculado como:

$\mathrm{N}_{\text {liberado }}=\left[\mathrm{Ni}_{\text {residuo }}-\mathrm{Ni}_{\text {testigo }}\right]_{\mathrm{t}}$ Donde Ni es la suma de $\mathrm{N}-\mathrm{NO}_{3}{ }^{-}+\mathrm{N}-\mathrm{NH}_{4}{ }^{+}$.

El $\mathrm{N}$ mineral neto $\left(\mathrm{N}_{\text {net }}\right)$ del residuo, se calculó como la diferencia entre $\mathrm{N}$ liberado en el tiempo " $t$ " y el cuantificado a la instalación $(\mathrm{t}=0$ ).

$\mathrm{El}$ porcentaje de $\mathrm{N}$ total liberado en el tiempo " $\mathrm{t}$ " se calculó como:

$\% \mathrm{~N}_{\text {net }}=\left[\left(\mathrm{N}_{\text {net }}\right) / \mathrm{N}_{\mathrm{o}}\right] * 100$

Donde $\mathrm{N}_{\mathrm{o}}$ es el $\mathrm{N}$ orgánico total del residuo agregado al inicio de la incubación.

\section{Análisis estadístico}

Todos los datos fueron sometidos al análisis de variancia (ANVA). Los promedios fueron comparados mediante la prueba de comparación de medias HSD (diferencia honestamente significativa) de Tukey, con un nivel de significación de 0.05. Los análisis fueron efectuados empleando los paquetes Agricolae, EPlot2, y $x x$ del ambiente para cómputo estadístico R versión 3.4.3 (R Core Team, 2017). 


\section{Resultados y Discusión}

\section{Liberación del nitrógeno mineral $\left(\mathrm{N}-\mathrm{NH}_{4}{ }^{+}+\right.$ $\left.\mathrm{N}-\mathrm{NO}_{3}{ }^{-}\right)$}

Las fluctuaciones de $\mathrm{N}$ mineral liberado de los cinco residuos incubados por 120 días se muestran en la Figura 1. Los dos estiércoles (EP y ER) y el LF se incrementaron hasta alcanzar su máximo pico de $\mathrm{N}$ mineralizado a los tres meses, a partir del cual disminuyeron hasta finalizar el periodo de incubación; el ERP mostró un comportamiento constante de mineralización y la HP registró incrementos hasta los 120 días. Estos comportamientos revelaron que los tres estiércoles (EP, ER y ERP) y el LF presentaron su máxima cantidad de $\mathrm{N}$ mineral liberado a los 90 días,

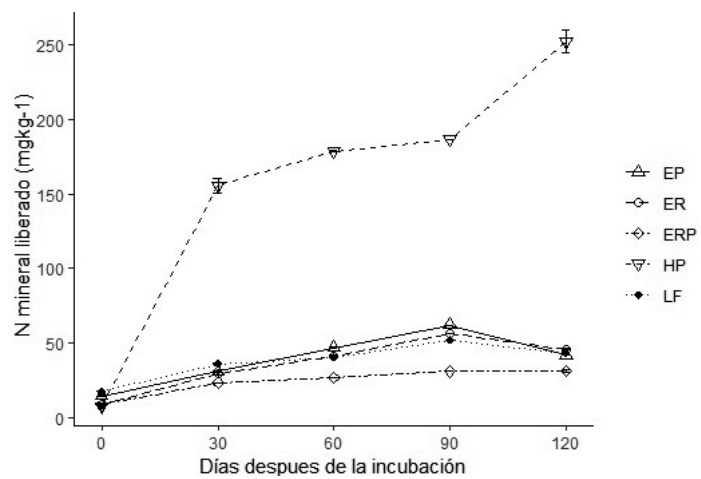

Figura 1. Fluctuaciones de $\mathrm{N}$ mineral $\left(\mathrm{N}-\mathrm{NH}_{4}{ }^{+}+\mathrm{N}^{-\mathrm{NO}_{3}}{ }^{-}, \mathrm{mg}\right.$ $\mathrm{kg}^{-1}$ ) liberado de cinco residuos avícolas: estiércol de gallina ponedora (EP), estiércol de gallina reproductora (ER), estiércol con restos de pollo (ERP), harina de pluma (HP) y lodo de flotación (LF), incubados en diferentes tiempos de incubación. mientras que la HP continuó mineralizándose hasta los 120 días.

El estiércol con restos de pollo (ERP) mostró las más bajas cantidades de $\mathrm{N}$ mineralizado, mientras que las registradas por el lodo de flotación (LF) y los otros dos estiércoles (ER y EP) fueron mayores a esta, pero similares entre sí. Las cantidades liberadas por la harina de pluma sobrepasaron a los demás residuos durante todo el periodo de incubación, tanto es así que a los 120 días liberó cinco, siete y diez veces las cantidades máximas de los estiércoles (EP y ER), LF y ERP, respectivamente. Las grandes cantidades mineralizadas pueden ser explicadas por los altos porcentajes de proteína cruda (82\%), 15,1\% de N y un alto porcentaje de material volátil $(82 \%)$ en su composición (Tesfaye et al., 2017).

Los datos de $\mathrm{N}$ mineral neto no revelan inmovilización en los residuos (Tabla 2). Sin embargo, debido a que las evaluaciones fueron hechas cada 30 días, podría haber ocurrido una mineralización neta e inmovilización alternada dentro de cada intervalo de evaluación y, por lo tanto, nuestros datos mensuales no podrían reflejar un verdadero juicio de la mineralización. Por ejemplo, se observaron procesos alternados de mineralización neta e inmovilización, durante cortos periodos de tiempo, a lo largo de 120 días de incubación (Azeez y Van, 2010). Li y Li (2014) reportaron inmovilización neta en los suelos mezclados con estiércol de aves antes de los 28 días de incubación.

La dinámica de mineralización de nitrógeno en la HP consta de tres fases marcadas, dentro de las

Tabla 2. Contenido de $\mathrm{N}$ mineral neto $\left(\mathrm{N}-\mathrm{NH}_{4}{ }^{+}+\mathrm{N}^{-\mathrm{NO}_{3}}{ }^{-}\right)$ en arena con residuos avícolas a través del periodo de incubación.

\begin{tabular}{lcccc}
\hline \multirow{2}{*}{\multicolumn{1}{c}{ Residuo }} & \multicolumn{4}{c}{ Tiempo de incubación (d) } \\
\cline { 2 - 5 } & 30 & 60 & 90 & 120 \\
\cline { 2 - 5 } & \multicolumn{4}{c}{${\mathrm{N}-\mathrm{NH}_{4}{ }^{+}+\mathrm{N}^{-} \mathrm{NO}_{3}{ }^{-}\left(\mathrm{mg} \mathrm{kg}^{-1}\right)}$} \\
\cline { 2 - 5 } Estiércol de ponedoras & $16,4 \mathrm{~b}$ & $32,3 \mathrm{~b}$ & $47,6 \mathrm{~b}$ & $27,6 \mathrm{bc}$ \\
Estiércol de reproductoras & $20,3 \mathrm{~b}$ & $32,3 \mathrm{~b}$ & $47,0 \mathrm{c}$ & $36,7 \mathrm{~b}$ \\
Estiércol con restos de pollo & $14,9 \mathrm{~b}$ & $18,2 \mathrm{~d}$ & $22,6 \mathrm{e}$ & $22,7 \mathrm{c}$ \\
Harina de pluma & $148,2 \mathrm{a}$ & $171,4 \mathrm{a}$ & $179,2 \mathrm{a}$ & $245,1 \mathrm{a}$ \\
Lodo de flotación & $18,3 \mathrm{~b}$ & $23,0 \mathrm{c}$ & $34,4 \mathrm{~d}$ & $25,5 \mathrm{bc}$ \\
Nivel de significación & $* * *$ & $* * *$ & $* * *$ & $* * *$ \\
\hline
\end{tabular}

Los valores dentro de cada columna seguidos por la misma letra no son significativamente diferentes a $\mathrm{P}<0,05$, de acuerdo a la prueba de comparación de medias HSD de Tukey. Niveles de significación: 'n.s.' $>0,05$, '*' $<0,05$, '**' $<0,01$, '****' $<0,001$. 
cuales se muestran dos puntos de incremento brusco de $\mathrm{N}$ mineral. En la primera fase, se observó un incremento rápido hasta los 30 días; en la segunda (30 a 60 días) los aumentos fueron graduales y en la tercera (90 a 120 días) hubo nuevamente un rápido incremento. Por el contrario, la incubación de HP con un suelo agrícola a $30^{\circ} \mathrm{C}$, por 60 días, reportó sólo dos fases de mineralización con un punto de incremento brusco de $\mathrm{N}$ mineral. Dicho punto se registró a los 14 días después de la incubación, como resultado de las actividades de dos distintos grupos de microorganismos y enzimas: los que descomponen la harina de plumas (deshidrogenasas, proteasas) y los que degradan los productos secundarios de esa descomposición (bacterias proteolíticas, hidrolizadores de celulosa). La segunda fase consistió en un incremento gradual, producido por la mineralización del $\mathrm{N}$ retenido en la biomasa microbiana que se desarrolló mientras se descomponía la HP y los productos secundarios de dicha degradación (Hadas y Kautsky, 1994). Sin embargo, en nuestro estudio el material con el que se mezcló el residuo (HP) no fue un suelo agrícola (que está provisto de poblaciones de microorganismos y enzimas), sino un material inerte (arena), por lo que el tiempo tardío del primer incremento (30 días) en la mezcla de arena con HP podría deberse a una lenta proliferación por adaptación de los escasos microorganismos existentes en la arena. La razón de suponer la presencia de escasos microorganismos en la arena es que al evaluar el testigo (arena sin adición de residuo) se registró una cantidad marginal $\mathrm{N}$ mineral, la cual fue restada en cada tiempo de evaluación.

En el caso de los dos estiércoles (EP y ER) y el lodo de flotación (LF), el ritmo de mineralización

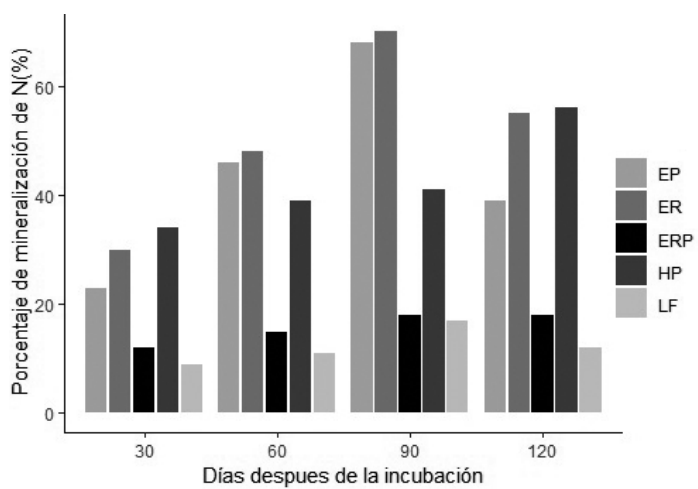

Figura 2. Porcentaje de N orgánico mineralizado de arena con cinco residuos avícolas en diferentes tiempos de incubación. está conformado también por tres etapas, con un primer incremento hasta los 30 días, seguido de un aumento gradual (30-90 días) hasta alcanzar su máximo a los 90 días y luego una disminución significativa hasta finalizar el periodo de incubación. El estiércol con restos de pollo (ERP) mostró un comportamiento dividido en dos fases: en la primera, un aumento hasta los 30 días y a partir de ahí, una constante mineralización hasta culminar la incubación. Li y Li (2014) observaron cuatro fases de mineralización durante la incubación de estiércol de aves mezclado con suelo agrícola: una fase inicial de liberación rápida (10-20 días), seguida de una fase lenta (30-40 días), una fase de mineralización máxima (55-90 días) y finalmente una fase de mineralización declinada (120 días). La liberación inicial rápida se debió principalmente al secado y humectación de los estiércoles (antes de la incubación), y posteriormente la mayor actividad de los microorganismos provocó una reducción del $\mathrm{N}$ disponible inmovilizándolo (etapa gradual). La máxima mineralización fue el resultado de la transformación de nitrógeno inmovilizado a $\mathrm{N}$ inorgánico que finalmente mostró tendencia a disminuir hasta el día 120 por aparentes procesos de volatilización. Contrariamente a la dinámica de mineralización de $\mathrm{N}$ de los estiércoles, un experimento de incubación por 120 días reportó inmovilización en los primeros 30 días, seguida de una rápida mineralización a los 40 días, pero posterior disminución, hasta el grado de inmovilización al finalizar el experimento (Azeez

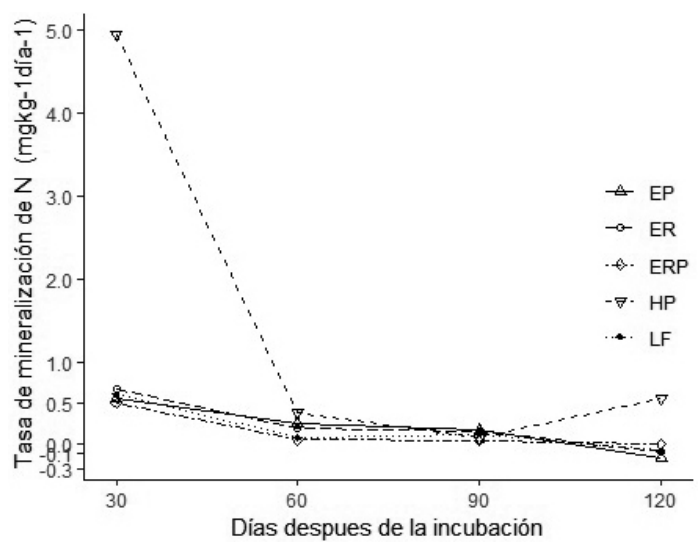

Figura 3. Tasa de mineralización de $\mathrm{N}\left(\mathrm{N}_{-} \mathrm{NH}_{4}{ }^{+}+\mathrm{N}^{-} \mathrm{NO}_{3}{ }^{-}, \mathrm{mg}\right.$ $\mathrm{kg}^{-1}$ día $\left.^{-1}\right)$ de arena con cinco residuos avícolas: estiércol de gallina ponedora (EP), estiércol de gallina reproductora (ER), estiércol con restos de pollo (ERP), harina de pluma (HP) y lodo de flotación (LF), incubados en diferentes tiempos de incubación. 
y Van, 2010). Este comportamiento se debió a que el estiércol fue mezclado con un suelo de textura franco arcillo arenoso (mientras que en este estudio se usó arena), el cual tuvo un efecto mayor en el proceso de retención de $\mathrm{N}$ mineral (amonio y nitrato) por coloides y en la disminución del porcentaje de aireación comparada con la mezcla de HP con arena, lo que produjo un mayor grado de inmovilización y menor mineralización. Investigaciones anteriores han demostrado que la mineralización de $\mathrm{N}$ en suelos de textura gruesa es más favorecida que en suelos de textura fina (Hassink et al., 1994).

El N mineralizado de los estiércoles y LF provino principalmente del $\mathrm{N}$ hidrolizable, especialmente de $\mathrm{N}^{-\mathrm{NH}_{4}}{ }^{+}$y N-aminoácido, siendo este último el que más contribuyó (Li y Li, 2014), mientras que la mineralización de la HP se vio favorecida, entre otros factores, por su composición química, anteriormente mencionada (Tesfaye et al., 2017). La proliferación de microbios en la etapa inicial podría haber conducido a competencias entre ellas por los nutrientes, seguido de mortandad, lo que repercutiría en la mineralización gradual durante la segunda fase (30-90 días). Posteriormente, la nutrición pudo verse afectada provocando un mayor grado de mortandad de los microbios y consecuente decrecimiento de la mineralización en los EP, ER y LF a los 120 días. En el caso de la HP, la descomposición de la biomasa muerta produjo la liberación de $\mathrm{N}$ y otros nutrientes microbianos, los que provocaron nuevamente una proliferación intensa de los pocos microorganismos tolerantes a la mortandad anteriormente registrada. Es por ello que en el periodo de 90 a 120 días la mineralización de $\mathrm{N}$ se incrementó.

La lenta y baja cantidad de $\mathrm{N}$ mineralizado del ERP, durante los 120 días, podría ser causa del mayor grado de compostaje al que fue sometido antes de ser tomado para el experimento de incubación. Esto coincide con lo encontrado en un experimento de incubación por 56 días a $25^{\circ} \mathrm{C}$, donde el estiércol altamente compostado mineralizó sólo el 5.8\% del $\mathrm{N}$ total, mientras que el estiércol medianamente compostado mineralizó el 40\%. Estos datos comprobaron que los estiércoles con mayor grado de compostaje tienden a liberar el $\mathrm{N}$ de forma lenta y en pocas cantidades (Masunga et al., 2016).

Si bien la HP liberó la mayor cantidad en mg $\mathrm{kg}^{-1}$ de $\mathrm{N}$ mineral, en términos de porcentajes, solo mineralizó el 56\% en su punto máximo (120 días), en tanto que los EP y ER mineralizaron hasta $70 \%$ cuando alcanzaron su máximo potencial de mineralización (90 días). El ERP mostró un comportamiento distinto a los otros estiércoles: mineralizó menos del $20 \%$ en su punto máximo, similar al LF. El porcentaje de mineralización en la HP en el experimento fue similar al reportado por Hartz y Johnstone (2006) en solo dos semanas de incubación en un suelo manejado con producción orgánica. El comportamiento más lento de la mineralización de la HP en nuestro experimento puede deberse al empleo de arena lavada como sustrato, que pudo retrasar la colonización microbiana necesaria para la descomposición de la queratina de las plumas (Lange et al., 2016). En otro estudio, un suelo agrícola con estiércol de aves liberó el $10 \%$ de $\mathrm{N}$ mineral en su punto máximo de los 55 hasta los 90 días después de la incubación, y posteriormente declinó hasta los 120 días (Abbasi et al., 2007). A través de los 120 días de incubación en el presente experimento, la liberación del nitrógeno en los residuos se ubicó en el siguiente orden: ER > EP > HP > ERP > $\mathrm{LF}$, con porcentajes de mineralización (promedio) de $50,44,42,16$ y $12 \%$ del $\mathrm{N}$ orgánico total, respectivamente.

Durante los tres primeros meses, el patrón de liberación de $\mathrm{N}$ mineral fue similar al de amonificación y distinto al de nitrificación. Esto indica que el $\mathrm{N}$ mineralizado durante este periodo se concentró más en amonio que en nitrato. En la última fase (90-120 días), la nitrificación de HP fue extremadamente creciente, y decreciente en la amonificación, de tal manera que el $\mathrm{N}$ mineral total se concentró más en las cantidades nitrificadas.

\section{Tasa de mineralización}

Las tasas de mineralización de $\mathrm{N}$ fueron mayores durante los 30 primeros días en todos los residuos, y fueron disminuyendo hasta el final del periodo de incubación, excepto en la HP, pues en el último periodo volvió a incrementarse, pero con menor intensidad que en la primera fase. Esto parece indicar que la HP se comporta como un fertilizante de liberación rápida más que moderada, y que la mayor parte del nitrógeno que contiene puede mineralizarse en cinco semanas (Choi y Nelson, 1996).

La relación $\mathrm{C} / \mathrm{N}$ en el EP y el ER fue mayor a 15 , pero no se evidenció inmovilización a lo largo 
del periodo de incubación. Esto no concuerda con lo reportado por Beauchamp y Paul (1989), quienes señalaron que el estiércol con una proporción de $\mathrm{C} / \mathrm{N}$ superior a 15 conduciría a la inmovilización neta de $\mathrm{N}$, mientras que las proporciones $\mathrm{C}$ : $\mathrm{N}$ total inferiores a 15 darían como resultado una mineralización neta de N. Qian y Schoenau (2002) encontraron que la aplicación de compost a un suelo, pese a su baja relación $\mathrm{C} / \mathrm{N}$, tuvo un comportamiento similar de mineralización de $\mathrm{N}$ que el control, lo que podría responder a la presencia de materiales recalcitrantes formados durante el proceso de compostaje. Esto concuerda con lo observado en nuestro estudio, puesto que el ERP estuvo altamente compostado y pese a su baja $\mathrm{C} / \mathrm{N}$, se mineralizó muy lentamente. Por lo tanto, se concluye que si bien la relación $\mathrm{C}$ : $\mathrm{N}$ es un factor importante que afecta la tasa de mineralización de las enmiendas orgánicas (Mueller et al., 1998), otras características, como el grado de degradación y polimerización, también son consideraciones relevantes.

\section{Dinámica del $\mathrm{N}-\mathrm{NH}_{4}{ }^{+}$y $\mathrm{N}-\mathrm{NO}_{3}{ }^{-}$}

Las cantidades de $\mathrm{N}^{-\mathrm{NH}_{4}}{ }^{+}$se incrementaron en todos los residuos hasta los 90 días (Figuras 4 y 5). Sin embargo, el aumento fue marcado durante los 30 primeros días en la harina de pluma. A partir de ahí el nitrógeno amoniacal fluctuó ligeramente hasta los 90 días, pero con cantidades mucho mayores a los demás residuos. Después de los 90

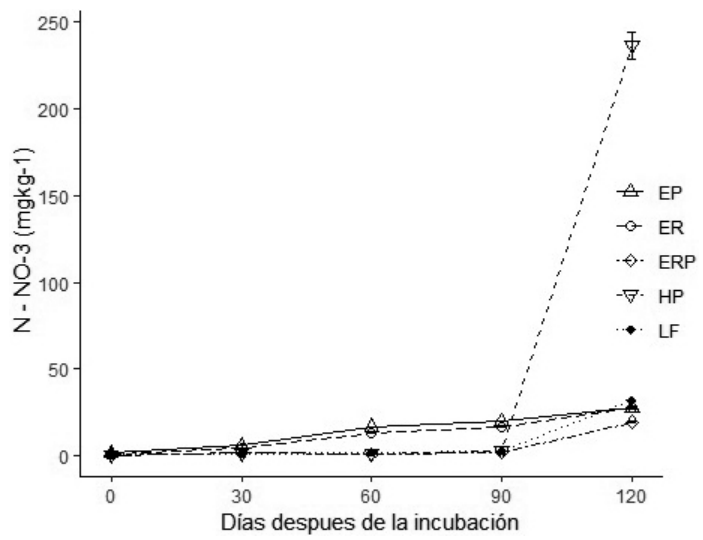

Figura 4. Fluctuaciones de $\mathrm{N}_{-} \mathrm{NO}_{3}{ }^{-}$liberado $\left(\mathrm{mg} \mathrm{kg}^{-1}\right)$ de cinco residuos avícolas: estiércol de gallina ponedora (EP), estiércol de gallina reproductora (ER), estiércol con restos de pollo (ERP), harina de pluma (HP) y lodo de flotación (LF), incubados en diferentes tiempos de incubación. días, la liberación de $\mathrm{N}-\mathrm{NH}_{4}{ }^{+}$declinó en los cinco residuos, pero de forma significativa en la harina de pluma. Contrariamente, la liberación de $\mathrm{N}^{-\mathrm{NO}_{3}}{ }^{-}$fue muy ligera, baja y constante hasta los 90 días en los cinco residuos. Desde entonces, la liberación de nitratos aumentó rápidamente, pero con gran ventaja en la harina de pluma. Los resultados indican que en los primeros 90 días predominó la amonificación del nitrógeno orgánico, mientras que la nitrificación fue muy escasa, situación que se revirtió en el último periodo de incubación (90120 días). Similarmente, al incubar HP por ocho semanas, se reportó liberación rápida de $\mathrm{N}-\mathrm{NH}_{4}{ }^{+}$ durante más de siete días perjudicando la formación de nitratos. Al parecer, la baja concentración de $\mathrm{O}_{2}$ en el medio como resultado de la rápida oxidación del material orgánico a amoniaco impidió la nitrificación (Hadas y Kautsky, 1994). Por otro lado, Li y Li (2014), en un estudio de mineralización de estiércol de aves, reportaron incremento rápido de amonio hasta los 14 días, seguido de un declive hasta los 161 días, mientras que las cantidades de nitrato aumentaron rápidamente durante los 56 a 86 días. La disminución del contenido de amonio a los 90 días puede explicarse por la inmovilización combinada con la nitrificación (que se incrementa en este periodo). Estos resultados difieren de lo encontrado en otro experimento de incubación de estiércol de aves, donde las cantidades de amonio durante los primeros 55 días resultaron insignificantes, mientras que las de nitrato fueron mayores (Azeez y Van Averbeke, 2010).

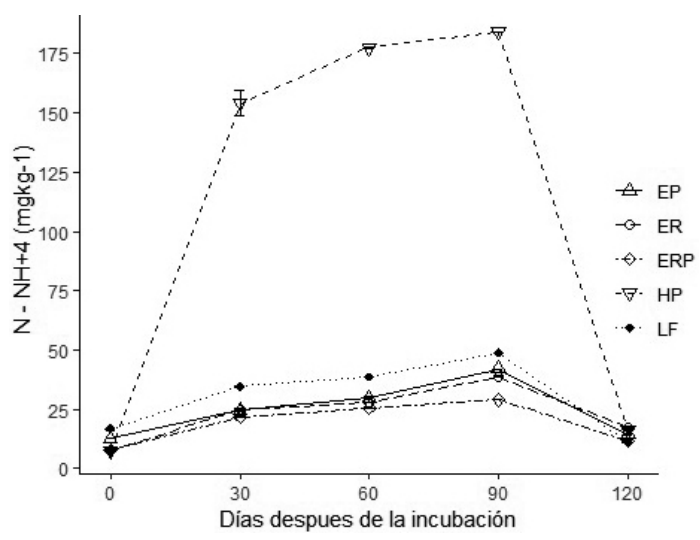

Figura 5. Fluctuaciones de $\mathrm{N}-\mathrm{NH}_{4}{ }^{+}$liberado $\left(\mathrm{mg} \mathrm{kg}^{-1}\right)$ de cinco residuos avícolas: estiércol de gallina ponedora (EP), estiércol de gallina reproductora (ER), estiércol con restos de pollo (ERP), harina de pluma (HP) y lodo de flotación (LF), incubados en diferentes tiempos de incubación. 


\section{Conclusiones}

Todos los residuos orgánicos investigados mostraron alto potencial como fuentes de $\mathrm{N}$ mineral; sin embargo, su comportamiento parece depender de su contenido de nitrógeno. La mineralización de $\mathrm{N}$ orgánico fue más alta en la harina de pluma, y alcanzó su mayor valor de $\mathrm{N}$ potencialmente mineralizable a los 120 días de incubación. Los demás residuos liberaron la máxima cantidad de $\mathrm{N}$ mineral a los 90 días. En todos los residuos, la máxima mineralización de amonio se observó a los 90 días, y de nitrato a los 120 días. Sin embargo, las tasas netas de mineralización fueron más altas durante los primeros 30 días de incubación, y el mayor valor se registró en la harina de plumas. De acuerdo a los presentes resultados, los estiércoles de gallina ponedora y reproductora, y la harina de pluma tienen mucho potencial para su uso en la elaboración de abonos orgánicos de rápida disponibilidad, mientras que el lodo de flotación puede ser empleado como fuente de liberación más lenta. Estudios más detallados de su comportamiento agronómico son requeridos para su uso en invernadero y campo.

\section{Agradecimientos}

Agradecemos al Programa Nacional de Innovación para la Competitividad y Productividad (Innóvate Perú) y a la Empresa Avícola San Fernando por el soporte financiero de la presente investigación mediante el proyecto Desarrollo de fertilizantes peletizados y/o granulados órganosminerales nutricionalmente óptimos para cultivos orgánicos emergentes (cacao, café, banano y palto). Contrato 250-PNICP-ITAI-2015- Innóvate Perú.

\section{Literatura Citada}

Abbasi, M.K.; Hina, M.; Khalique, A.; Khan, S.R.

2007. Mineralization of three organic manures used as nitrogen source in a soil incubated under laboratory conditions. Comm. Soil Sci. Plant Anal., 38: 1691-1711.

Abbasi, M.K.; Khaliq, A.

2016. Nitrogen mineralization of a loam soil supplemented with organic-inorganic amendments under laboratory incubation. Front. Plant Sci., 7: 1038.

Agehara, S.; Warncke, D.D.

2005. Soil moisture and temperature effects on nitrogen release from organic nitrogen sources. Soil Sci. Soc. Am. J., 69: 1844-1855.

Azeez, J.O.; Van Averbeke, W.

2010. Nitrogen mineralization potential of three animal manures applied on a sandy clay loam soil. Bioresour. Technol., 101: 5645-5651.

Beauchamp, E.G.; Paul J.W.

1989. A simple model to predict manure $\mathrm{N}$ availability to crops in the field. In: Hansen, J.A.;Henriksen, K. (eds). Nitrogen in organic wastes applied to soils. Harcourt Brace Jovanovich Publ. Boston, MA,US. pp. 140-149.

Cerrato, M.E.; Leblanc, H.A.; Kmeko, C.

2007. Potencial de mineralización de nitrógeno de bokashi, compost y lombricompost producidos en la Universidad EARTH. Tierra Tropical, 2: 183-197.

Choi, J-M.; Nelson, P.V.

1996. Developing a slow-release nitrogen fertilizer from organic sources: II. Using poultry feathers. J. Amer. Soc. Hort. Sci., 121(4): 634-638.

FiBL e IFOAM.

2019. The world of organic agriculture. Statistics and emerging trends 2019. Research Institute of Organic Agriculture (FiBL), IFOAM-Organic international. Alemania. 356 p.

Hadas, A.; Kautsky L.

1994. Feather meal, a semi-slow-release nitrogen fertilizer for organic farming. Fert. Res., 38: 165-170.
Hartz, T.K.; Johnstone P.R.

2006. Nitrogen availability from high-nitrogen containing organic fertilizers. Horttechnology, 16(1): 39-42.

Hassink., J.; Neutel, A.M.; De Rutter, P.C.

1994. C and N mineralization in sandy and loamy grassland soils: the role of microbes and microfauna. Soil Biol. Biochem., 26: 1565-1571.

Johnson, H.J.; Colquhoun, J.B.; Bussan, A.J.; Laboski, C.A.M. 2012. Estimating nitrogen mineralization of composted poultry manure, organic fertilizers, and green manure crops for organic sweet corn production on a sandy soil under laboratory conditions. Horttechnology, 22 (1): 37-43.

Lange, L.; Huang, Y.; Busk, P.K.

2016 Microbial decomposition of keratin in nature-a new hypothesis of industrial relevance. Appl. Microbiol. Biotechnol., 100: 2083-2096.

Li, L.L.; Li, S.T.

2014. Nitrogen mineralization from animal manures and its relation to organic $\mathrm{N}$ fractions. J. Integr. Agr., 13 (9): 2040-2048.

Masunga, R.M.; Uzokwe, V.N.; Mlay, P.D.; Odeh, I.; Singh,

A.; Buchan, D.; De Neve, S.

2016. Nitrogen mineralization dynamics of different valuable organic amendments commonly used in agriculture. Appl. Soil Ecol., 101: 185-193.

Mueller, T.; Jensen, L.S.; Nielsen, N.E.; Magid, J.

1998. Turnover of carbon and nitrogen in a sandy loam soil following incorporation of chopped maize plants, barley straw and blue grass in the field. Soil Biol. Biochem., 30: 561-571.

El Boushy, A.R.; van der Poel, A.F.B.; Walraven, O.E.D.

1990. Feather meal-A biological waste: Its processing and utilization as a feedstuff for poultry. Biol. Wastes, 32(1): 39-74.

Qian, P.; Schoenau, J.J.

2002. Availability of nitrogen in solid manure amendments with different C: N ratios. Can. J. Soil Sci., 82: 219-225. 
R Core Team.

2017. R: A Language and Environment for Statistical Computing. R Foundation for Statistical Computing, Vienna, Austria. Disponible en: https://www.R-project. org.

Tesfaye, T.; Sithole, B.; Ramjugernath, D.; Chunilall, V. 2017. Valorisation of chicken feathers: Characterisation of chemical properties. Waste Manag., 68: 626-635.
Tyson, S.C.; Cabrera M.L.

1993. Nitrogen mineralization in soils amended with composted and uncomposted poultry litter. Comm. Soil Sci. Plant Anal., 24: 2361-2374.

Yuen, S.H.; Pollard, A.G.

1953. Determination of nitrogen in soil and plant materials: Use of boric acid in the microkjeldahl method. J. Sci. Food Agr., 4(10): 490-496. 
